\title{
Disorder-Induced Light Confinement in Photonic Crystals as a Platform for Efficient Optical Sensing
}

\author{
Oliver J. Trojak*, Tom Crane*, Luca Sapienza \\ Department of Physics and Astronomy, University of Southampton, SO17 1BJ, Southampton, United Kingdom \\ equal contributors \\ Author e-mail address: l.sapienza@soton.ac.uk
}

\begin{abstract}
We demonstrate optical sensing with disorder-induced confined light on silicon nitride photonic crystal waveguides. For a refractive index change of $\approx 0.38$, we measure $14 \mathrm{~nm}$ wavelength shifts of an optical resonance $0.4 \mathrm{~nm}$ broad.

OCIS codes: (350.4238) Nanophotonics and photonic crystals; (280.4788) Optical sensing and sensors;

(300.6280) Spectroscopy, fluorescence and luminescence
\end{abstract}

\section{Introduction}

Optical sensing is of preeminent importance for a variety of applications [1]: it can enable detection of harmful or desired contaminants, it can confirm that expected reactions have taken place and can be used for quantitative analysis of the processes under study.

To this end, several kind of devices have been developed; in particular they have been based on plasmonic resonances [2] that have the advantage of relatively easy production and high yield, and photonic crystal cavities [3] that posses much sharper spectral resonances (and therefore higher sensitivity), but suffer from low scalability due to the highly engineered fabrication process required.

To overcome this issue, we follow a different approach and show that multiple scattering on unavoidable fabrication imperfections can be used as a platform for efficient light confinement in disordered photonic crystal waveguides. By using imperfections as a resource, we show that several high-quality optical cavities spontaneously appear along a fabricated photonic crystal waveguide operating at room temperature [4]. Such an approach represents a route for scalable, high quality optical sensors.

\section{Discussion of the results}

Silicon nitride suspended photonic crystal waveguides are fabricated and characterized by means of confocal photoluminescence spectroscopy, under $407 \mathrm{~nm}$ continuous-wave laser excitation. The emitted signal due to the intrinsic silicon nitride luminescence is collected by a confocal microscope and spectrally analyzed with a grating spectrometer equipped with a silicon charge-coupled device (see Fig.1a). Sharp spectral resonances, a signature of light confinement by disorder, are visible in the spectra collected when moving the excitation/collection spot along the waveguides [4].

(a)

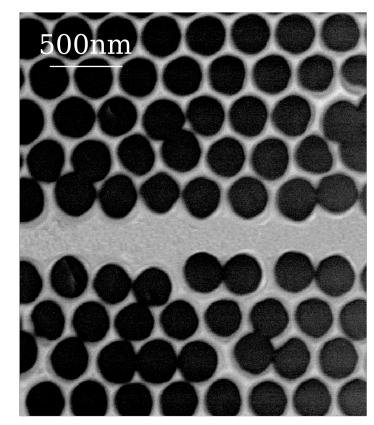

(b)

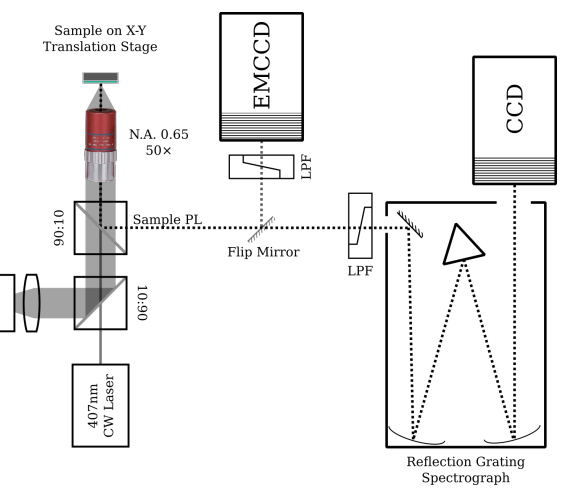

Figure 1: (a) Scanning electron micrograph of a suspended photonic crystal waveguide where disorder is introduced by displacing the three rows of holes on either side of the waveguide. (b) A confocal micro-photoluminescence (micro-PL) set up, an objective with Numerical Aperture (N.A.) of 0.65 is used to image and excite the sample, held on a xy-translation stage, with a 940-nm Light Emitting Diode (LED) or a Continuous Wave (CW) 407-nm laser respectively. The photoluminescence is either imaged on an Electron Multiplied Charge Coupled Device (EMCCD) or sent to a spectrometer for spectral characterization, after removing the excitation light with a Long Pass Filter (LPF). 
(a)

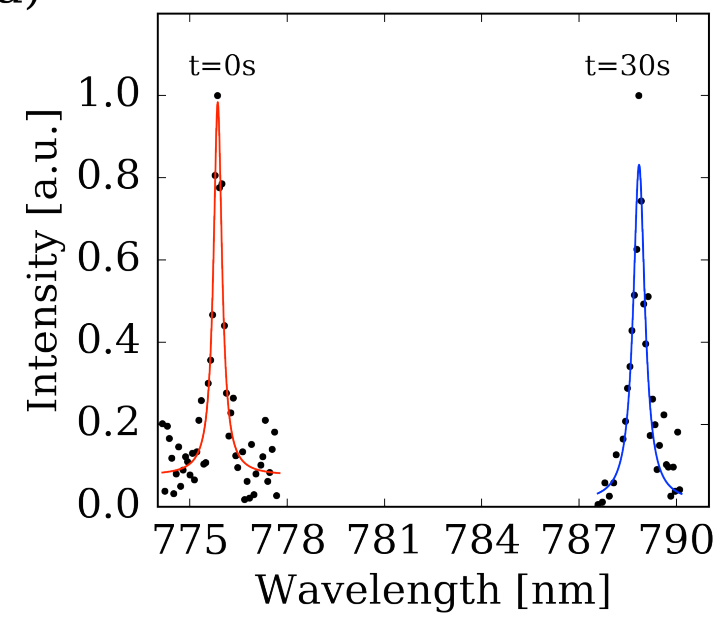

(b)

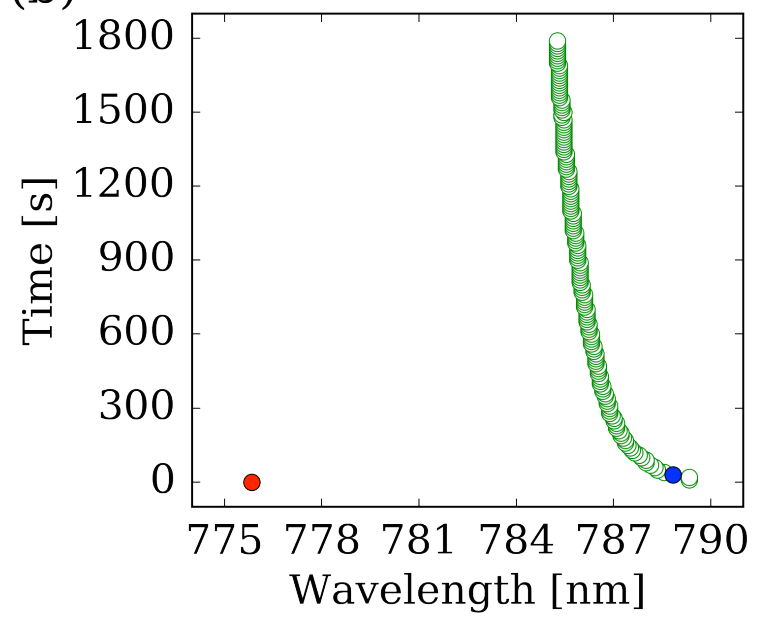

Figure 2: (a) Example of spectra (symbols) collected under a laser excitation power density of $28 \mathrm{~kW} / \mathrm{cm}^{2}$, at room temperature, and their Lorentzian fits (solid lines) collected before isopropyl alcohol is applied to the photonic crystal waveguide ( $\mathrm{t}=0$ ) and $30 \mathrm{~s}$ after isopropyl alcohol deposition. (b) Wavelength of the optical resonances plotted as a function of time, as obtained from the Lorentzian fits of the photoluminescence spectra (symbols, the error bars are too small to be visible) showing the time evolution of the process. The red and blue symbols correspond to the spectra in panel (a).

In order to characterize the response of our device to the presence of contaminants, we deposit an amount (estimated to be $\approx 20 \mathrm{p} \ell$ on the photonic crystal waveguide area of approximately $10^{3} \mu \mathrm{m}^{2}$ ) of isopropyl alcohol (with refractive index 1.38) on the sample's surface and monitor the emission wavelength of selected resonances throughout the process. When the contaminant is deposited on the sample, producing a local refractive index change of $\approx 0.38$, we observe a spectral shift of $14 \mathrm{~nm}$ of a cavity resonance with a full width half maximum of $0.4 \mathrm{~nm}$ (see Fig. 2).

The process is reversible: when the contaminant evaporates, the resonance shifts back after a few hours (the position of the resonance in the first 30 min after deposition is shown in Fig.2b). A calibration of the system would thus allow not only the verification of the presence of a contaminant but also the evaluation of its quantity and/or its refractive index.

\section{Conclusions}

We have demonstrated optical sensing with disorder-induced optical resonances, showing wavelength shifts of 35 times the linewidth of the optical cavity for a modest change in refractive index $(\approx 0.38)$, in a device operating at room temperature. By making use of fabrication imperfections, one can obtain tens of high-quality optical resonances along a $100 \mu \mathrm{m}$ long waveguide, without the need for highly engineered designs or iterative fabrication processes. Such an approach thus represents a novel route for scalable, room temperature, highly efficient optical sensors.

\section{References}

[1] J. Hodgkinson, R.P. Tatam, Optical gas sensing: a review, Measurement Science and Technology 24, 012004 (2013).

[2] M. Li, S.K. Cushing, N. Wu, Plasmon-enhanced optical sensors: a review, Analyst 140, 386 (2015).

[3] Y. Zhanga, Y. Zhaoa, R-Q. Lv, A review for optical sensors based on photonic crystal cavities, Sensors and Actuators A 233, 374 (2015).

[4] T. Crane, O.J. Trojak, L. Sapienza, Anderson localisation of visible light on a nanophotonic chip, https://arxiv.org/abs/1605.08614v2 (2016). 